\title{
A case of HIV, leprosy co-infection - presenting as immune reconstitution inflammatory syndrome
}

\section{Romita Bachaspatimayum, Bishurul Hafi}

\author{
Regional Institute of Medical Sciences, Imphal, Manipur, India
}

Corresponding author: Dr. Romita Bachaspatimayum, E-mail: romi1bac@gmail.com

\begin{abstract}
Though leprosy was officially declared eliminated in India, new cases continue to be reported. Introduction of Highly Active Antiretroviral Therapy (HAART) for Human Immunodeficiency Virus (HIV) infection in 1996, caused emergence of a new condition called Immune Reconstitution Inflammatory Syndrome (IRIS), which is the paradoxical deterioration of clinical status following HAART initiation. A 49 years old male patient who came with the complaints of dry skin and numbness of limbs 3 weeks after starting HAART was found to have IRIS syndrome and started on (MB- MDT) after which he developed type 1 lepra reaction. He was managed with systemic steroids with prompt recovery. Case is being reported to create awareness of this condition as more and more number of patients may be put on HAART due to increasing HIV prevalence in the region which may in turn lead to increase incidence of IRIS.
\end{abstract}

Key words: Leprosy; HAART; IRIS; AIDS

\section{INTRODUCTION}

Leprosy has been a major public health problem in many developing countries for centuries. Though it was officially declared eliminated in India since December 2005, new cases have been reported with varying prevalence throughout the nation. Introduction of Highly Active Antiretroviral Therapy (HAART) in 1996, caused emergence of a new condition called Immune Reconstitution Inflammatory Syndrome (IRIS), which is the paradoxical deterioration of clinical status following HAART initiation [1]. First case of leprosy or its reaction as IRIS syndrome was reported in 2003 and there are not much case reports as well as etiolopathological investigatory studies in the subject so far.

\section{CASE REPORT}

A 49 yr old paramilitary officer on irregular HAART for 10 months was referred from Antiretroviral therapy (ART) clinic, for numbness and gradually progressive, non itchy, painless, dry skin lesions over the forearms and legs. There was swelling and pain of feet for one week. He was started on HAART with Zidovudine, Lamivudine and Nevirapine but was interrupted due to skin rashes. He was shifted to Efavirenz subsequently and he had taken HAART for an effective period of seven months before presenting to us. He was on oral hypoglycemic agents as well as twice daily clotrimoxazole for eight months.

On examination, approximately 10 large dry wrinkled, mildly shiny and scaly hyperpigmented plaques of varied morphology and imperceptibly merged margins was detected over forearms and legs (Fig. 1). Dry irregular scaly plaques were seen on gluteal region, while annular reddish yellow plaque with central clearing and mildly raised peripheral rim was seen on left mammary area; ill defined plaques over lower back were present (Fig. 2). Sensations were diminished in all skin lesions in varying degrees. Muscle power was normal, great auricular, radial-superficial and deep, ulnar nerves were enlarged but non tender. Common peroneal nerves were enlarged and mildly tender bilaterally.

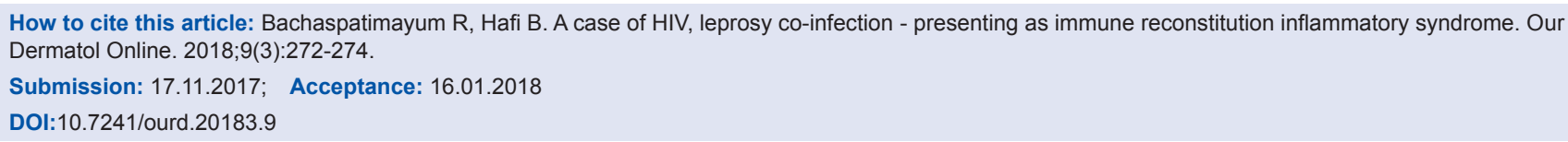


Hemogram was normal except for high ESR $\left(110 \mathrm{~mm} / \mathrm{l}^{\text {st }} \mathrm{hr}\right)$. RBS, LFT, KFT, Urine R/E, Thyroid profile, G6PD levels, Hepatitis serology, chest X-ray were all in normal limits. CD4 counts were 147/cumm and 161 /cumm before starting HAART and at the time of presentation respectively. Skin biopsy from the lesion on back showed mildly flattened epidermis, mild to moderate lymphocytic infiltration in papillary dermis with well formed epithelioid cell granulomas, peri-appendageal granuloma, lepra stain showed few acid fast bacilli; features which were consistent with diagnosis of Hansen's disease (BT type) (Fig. 3). Multibacillary multidrug therapy (MB-MDT) was started along with HAART.

Three weeks later he presented with tingling and numbness in whole body, pain over the thighs and arms for ten days and painful swelling of hands and feet for one wk. Increase in the size of existing skin lesions was noted. There was edema over bilateral hands and feet, claw hand was present. Ulnar, Common peroneal, Posterior Tibial nerves were thickened and tender. Paralysis was seen in small muscles of hands and foot. Diagnosis of type 1 lepra reaction was made and managed with oral Prednisolone $60 \mathrm{mg}$ /day. MBMDT and HAART continued. He was lost to followup further. Prior to the study, patient gave written consent to the examination and biopsy after having been informed about the procedure.

\section{DISCUSSION}

Immune Reconstitution Inflammatory Syndrome (IRIS) is defined as the occurrence or worsening of clinical and/ or laboratory parameters despite favorable outcome in HIV surrogate markers (CD4 counts and viral loads) [1]. It was originally reported in mycobacterium avium complex in 1998 [2]. Subsequently many opportunistic infections like herpes zoster, warts, hepatitis, cryptococcosis, histoplasmosis, toxoplasmosis, pneumocystis carinii infections as well as non infectious conditions like sarcoidosis, various autoimmune and neoplastic disorders were reported. IRIS can occur even among HIV negative individuals with sudden recovery of neutrophil counts after stopping chemotherapy or long term immunosuppressive therapy.

First case of leprosy associated with IRIS syndrome was reported on 2003 only, by Lawn et al. [3]. HIV infection has not been reported to increase susceptibility to leprosy infection markedly or alter the pathological course of the disease. A retrospective study on 4025

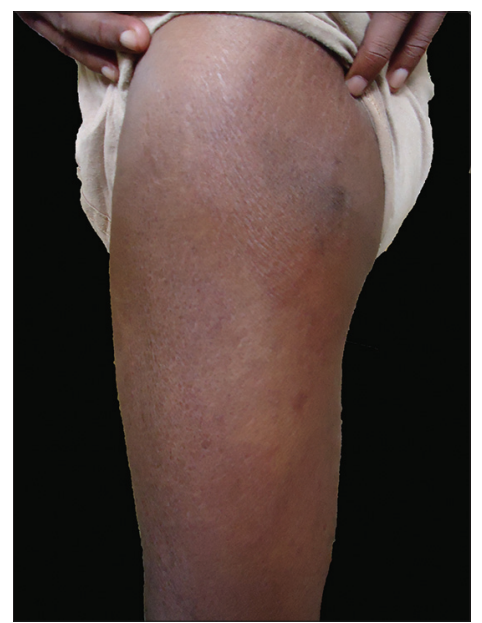

Figure 1: Shiny scaly plaque over lateral aspect of thigh.

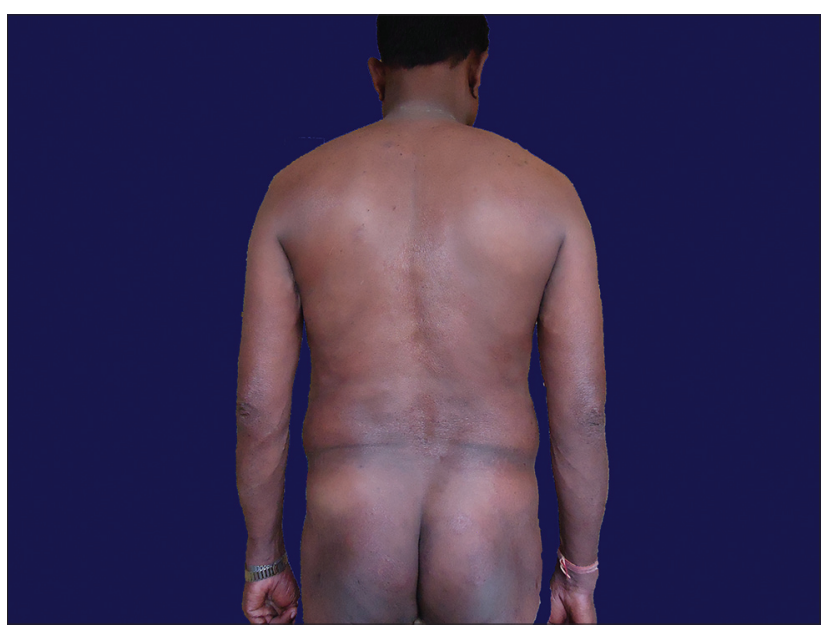

Figure 2: Shiny scaly plaques with imperceptively merged margins over back and gluteal region.

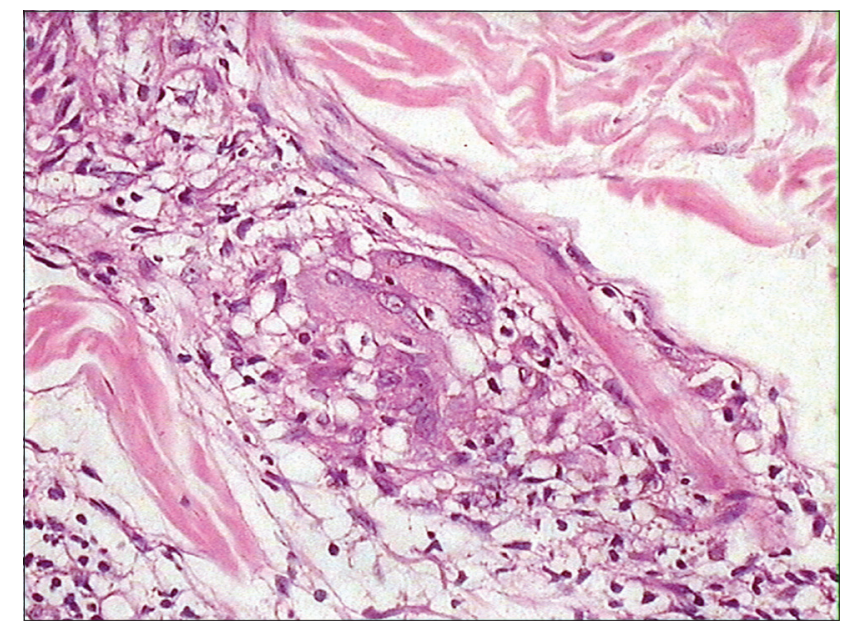

Figure 3: H.P.E (H\&E, 10x) showing papillary dermis with mild to moderate lymphocytic infiltration and well formed epithelioid cell granulomas.

leprosy patients in India described only 5 cases of coinfection with HIV [4]. Similarly, another common 
belief that HIV, by decreasing immunity may lead to increased incidence of lepromatous cases, also was rejected by systematic studies. Tuberculoid cases found to be favored in co-infection with HIV. In a cohort study conducted by Euzenir et al showed HIV infection is preferentially associated with the emergence of previously undiagnosed BT/PB leprosy. It also showed an improved immune status, a higher CD4+ counts and lower viral loads favors diagnosis of leprosy in HIV co-infected patients. This observation provides support for the reports suggesting leprosy as part of IRIS [5].

Though IRIS is a well discussed entity, an universally acceptable diagnostic criteria is still lacking. According to the proposal by Deps and Lockwood in 2010 diagnosis can be achieved from the following; 1) leprosy and/or type 1 reaction presenting within six months of starting HAART 2) advanced HIV infection 3) low CD4+ count before start of HAART and 4) CD4+ count increasing after HAART has been started [6]. Ideally, both viral load and CD4+ cell count should be used as diagnostic criteria. If data on viral load is not available, then should be an increase in CD+ count associated with starting HAART [7]. In our case, there is only a minimal increase in CD4+ count after starting HAART and viral load was not done due to un affordability. It has been found that even a minimal decrease in viral load in the absence of a significant rise in CD4+ cell count itself can precipitate IRIS [8]. However viral load was not tested in our patient before initiation of HAART due to non availability during that period and NACO (National AIDS Control Organization) consider it to be an optional test.

\section{CONCLUSION}

In HIV infected patients with low CD4+ counts $<200 / \mathrm{cmm}$, on HAART, if skin lesions with numbness appearing within first six months of initiation of the treatment, a differential diagnosis of leprosy in the form of immune reconstitution inflammatory syndrome should be made with priority in high endemic areas. A wide variety of atypical clinical presentations and serious reactions should be anticipated. More systematic studies are required to understand the immune and inflammatory mechanisms associated with the condition.

\section{CONSENT}

The examination of the patient was conducted according to the Declaration of Helsinki principles.

\section{REFERENCES}

1. Bosaniya SS. The immune reconstitution inflammatory syndrome. Indian J Dermatol. 2011;56:476-79.

2. Shelburne SA, Montes M, Hamill RJ. Immune reconstitution inflammatory syndrome: More answers, more questions. J Antimicrob Chemother. 2006;57:167-70.

3. Lawn SD, Wood C, Lockwood DN. Borderline tuberculoid leprosy: An immune reconstitution phenomenon in a human immunodeficiency virus infected person. Clin Infect Dis. 2003;36:e5-6.

4. Hussain T, Kulshreshta KK, Ghei SK, Natarajan M, Katoch K, Sengupta U. HIV seroprevalance in leprosy patients. Int J Lepr Other Mycobact Dis. 2000;68:67-9.

5. Sarno EN, Illarramendi X, Nery JC, Galhardo MG, Penna MF, Sampaio EP, et al. HIV-M.Leprae interaction: can HAART modify the course of Leprosy. Public Health Reports. 2008;123:206-12.

6. Deps P, Lockwood DN. Leprosy presenting as Immune Reconstitution Inflammatory Syndrome- proposed definitions and classifications. Lepr Rev. 2010;81:59-68.

7. Sanghi S, Grewal RS, Vasudevan B, Lodha N. Immune reconstitution inflammatory syndrome in leprosy. Indian J Lepr. 2011;83:61-70.

8. Hirsch HH, Kaufmann G, Sendi P, Battegay M. Immune reconstitution in HIV infected patients. Clin Infect Dis. 2004;38:1159-66.

Copyright by Romita Bachaspatimayum, et al. This is an open-access article distributed under the terms of the Creative Commons Attribution License, which permits unrestricted use, distribution, and reproduction in any medium, provided the original author and source are credited. Source of Support: Nil, Conflict of Interest: None declared. 